\title{
Enhancement of the ASDEX Upgrade Real-Time Plasma Position Reflectometry Diagnostic
}

\author{
J. M. Santos, G. S. Santos, M.Zilker, L.Guimarãis, C.Rapson, W.Treutterer, B. Gonçalves, \\ and the ASDEX Upgrade Team
}

\begin{abstract}
Microwave O-mode reflectometry is a diagnostic technique that will play an important supplementary role for plasma position control for ITER and forseeably for DEMO. Density profiles from reflectometry will provide, at high temporal resolutions, estimates of the gap between the plasma magnetic separatrix and the tokamak vessel walls. These estimates will be used to detect and correct drifts in the magnetic gap measurements, the primary measurements used for plasma position and shape control. The feasibility of this alternative feedback control approach was demonstrated in 2011 on ASDEX Upgrade (AUG) [1], where the reflectometry gap estimate actually replaced the corresponding magnetic measurement in the position control loop. Presently, the AUG's real-time (RT) reflectometry diagnostic is being upgraded to improve not only its density range coverage but also its acquisition and RT data processing performance. The diagnostic is now capable of acquiring a total of $\mathbf{1 6}$ channels (previously 8) from which 8 , corresponding to microwave bands $\mathrm{K}, \mathrm{Ka}, \mathrm{Q}$ and $\mathrm{V}$ from both the high (HFS) and low field side (LFS) reflectometers, are used in the RT density profile and separatrix gap calculations. The modern NUMA hardware architecture of the updated data processing server allows for an efficient and separate handling of the data-flows produced by the hosted acquisition systems. The higher RAM and CPU interconnection bandwidths allow the implementation of new operation modes that exploit the very high data throughput $(>1.2 \mathrm{~GB} / \mathrm{s}$ ) of each of the two used acquisition boards. RT reconstruction of the density profiles is a complex algorithm, whose performance will also be improved by the additional processing power (16 cores instead of 8). The system was specified to acquire and store HFS and LFS density profile data every $<250 \mu$ s whilst simultaneously producing profile measurements for RT control every $1 \mathrm{~ms}$. In this new operation mode, identical to the one planned for the ITER plasma position reflectometer (PPR), the diagnostic can reach an inbound data throughput (ADCs to RT processing host) and a computational load that are closer to the ones expected to be generated by each of the planned 4 ITER PPRs $(\approx 3$ GB/s maximum inbound and outbound data stream bandwidth per PPR). Herein we discuss the enhancements introduced in the AUG's RT reflectometry diagnostic to implement the new operation mode and perform the aimed control experiments. Preliminary RT experimental data obtained in both the HFS and LFS is shown to illustrate the system's plasma position and shape control capabilities to be demonstrated during AUG's 2014 experimental campaign.
\end{abstract}

Index Terms-Real-time reflectometry, plasma position control, high throughput data acquisition, real-time data processing, ASDEX Upgrade, ITER control diagnostics.

J. M. Santos, G. S. Santos, L. Guimarãis and B. Gonçalves are with the Instituto de Plasmas e Fusão Nuclear, Instituto Superior Técnico, Universidade de Lisboa, 1049-001 Lisboa, Portugal telephone: +351.218419080, e-mail: jsantos@ipfn.ist.utl.pt).

M. Zilker, W. Treutterer, C. Rapson and the ASDEX Upgrade Team are with Max-Planck-Institut für Plasmaphysik, 85748 Garching, Germany.

Manuscript received May 28, 2010; revised XXXXXX xx, 2010.

\section{INTRODUCTION}

$\mathbf{I}$ $\mathrm{N}$ existing Fusion experimental devices, like the midsized ASDEX Upgrade (AUG) tokamak, the control of the hot plasma column position and shape is performed by means of magnetic actuators (magnetic field coils) and the feedback of plasma-wall gaps calculated using magnetic sensor (pickup coils) readings. In future fusion reactors, operated continuously or in long pulses ( $>1000 \mathrm{~s}$ on ITER), these measurements may be affected by drifting integrators or radiation induced voltages in the magnetic pickup coils. As the feedback control of the plasma position plays a vital role for machine protection and disruption avoidance, alternative non-magnetic measurements of the plasma boundary location are needed to supplement or correct the magnetic measurements. O-mode reflectometry density profile measurements can be used to produce accurate location estimates of specific edge density layers with respect to a physical location near the vessel wall (plasma-wall gaps). Such estimates rely solely on time-offlight measurements which are independent of the measurement history and are robust against the systematic errors which affect magnetic diagnostics. Furthermore, to avoid radiation damage, on large sized devices such as ITER, DEMO and future reactors, magnetic pickup coils will need to be placed far from the plasma what will contribute to lower both sensor sensitivity and measurement rate. O-mode reflectometers, on the other hand, can endure the harsh radiation environment of such machines whilst retaining all the required control measurement capabilities, namely accuracy $(<1 \mathrm{~cm})$ and time resolution $(<1 \mathrm{~ms})$.

The feasibility of this alternative feedback control approach was first demonstrated in 2011 on ASDEX Upgrade (AUG) [1], where reflectometry gap estimates of the outermost lowfield side (LFS), $R_{\text {out }}^{\prime}$, magnetic separatrix location were used to replace the corresponding magnetic measurement in the position control loop. On AUG, however, two O-mode reflectometers probe simultaneously the plasma along quasiequatorial lines-of-sight from the high (HFS) and low-field sides (LFS). With this diagnostic setup (see Fig. 1) it is possible to also produce, for plasma shape/position control, the innermost separatrix position estimate, $R_{i n}^{\prime}$, or naïve approximations for the geometric radius, $R_{\text {geo }}^{\prime}=\left(R_{\text {out }}^{\prime}+R_{\text {in }}^{\prime}\right) / 2$, and for the plasma minor radius, $a^{\prime}=\left(R_{\text {out }}^{\prime}-R_{\text {in }}^{\prime}\right) / 2$.

The demonstration on AUG of this extended control application is of the utmost importance for the design of ITER's plasma position reflectometers (PPR). Presently this supplementary control system is in the advanced requirement revision 

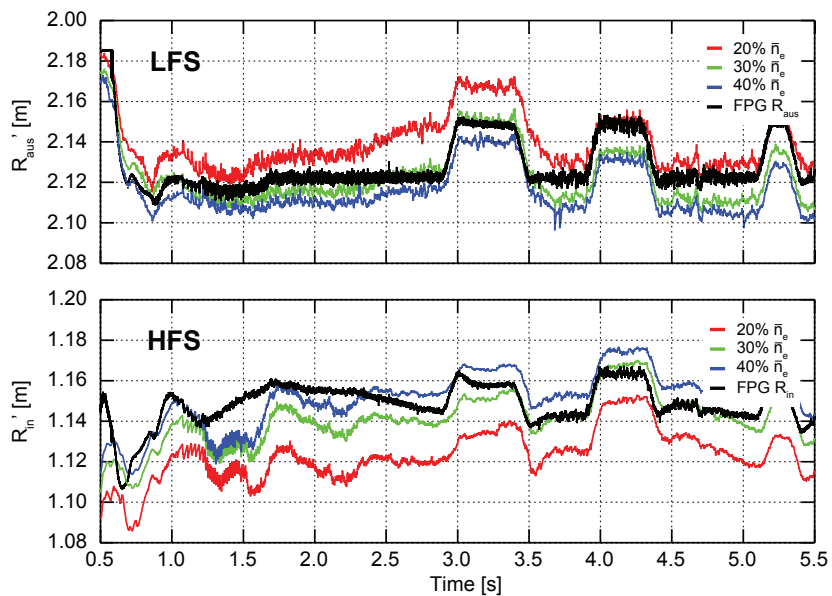

(a)

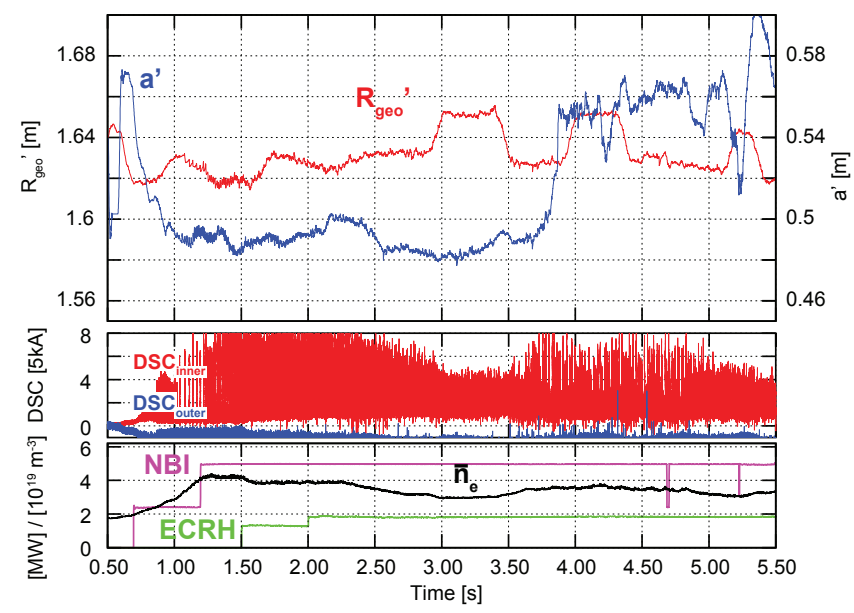

(b)

Fig. 1. Preliminary results obtained with the upgraded system in an $\mathrm{H}$ mode discharge (\#30660): (a) $R_{\text {in }}^{\prime}$ and $R_{\text {out }}^{\prime}$ for different separatrix density scalings, 20,30 and $40 \%$ of $\bar{n}_{e}$ (magnetic separatrix in black), (b) naïve approximations $R_{g e o}^{\prime}$ and $a^{\prime}$ corresponding to a $30 \% \bar{n}_{e}$ position estimate.

and definition phase. This means that its final configuration has not yet been fully established and can highly benefit from the added experience gained on AUG's control experiments. In fact, AUG's O-mode reflectometry system [2] has the unique characteristic of being able to probe the plasma along two of the four lines of sight foreseen for the future ITER's PPRs [3] (Fig. 2 inset). Although its microwave sources will be swept much faster than the ones in the now 20 year old AUG reflectometry system (probably $5 \mu \mathrm{s}$ vs AUG's $25 \mu \mathrm{s}$ ), both diagnostics will cover a very similar density range (a 15-75 GHz operating range has been proposed for ITER's PPRs).

Due to AUG's reflectometry diagnostic arrangement and to the flexibility and sophistication of the AUG discharge control system (DCS), it was decided to further pursue the validation of this new control application. The diagnostic's real-time (RT) data acquisition and processing system [4], custom built to perform the demonstration in 2011, was recently upgraded to allow the acquisition of an increased number of reflectometry channels, and a RT operation mode similar to the one proposed for ITER's PPR. Having to handle in RT a much increased data volume, whilst retaining the same control measurement data rate, the new system provides an adequate experimental setup in which to test and validate implementation solutions for the future ITER PPR fast plant system controller (FPSC). In the next sections we will describe the AUG PPR, how it compares with the present specification for the ITER PPR and in what ways the upgrades introduced in the AUG PPR improved its performance and capabilities, aiming at increasing the system's availability and reliability.

\section{Plasma POSITION CONTROL AND IMPROVED MEASUREMENT MODE}

On ASDEX Upgrade, a fully digital discharge control system (DCS) [5] is responsible for the control and monitoring of several physics quantities. This complex but flexible control system is fed by an increasing network of powerful RT diagnostics, which partially offload the main DCS from the heavy RT computations required to derive the control relevant physics parameters to be monitored and fed to its various multivariable feedback control loops. The herein described RT plasma position reflectometer is one such diagnostic, acquiring, at high acquisition rates (40 MSPS), bursts of raw data from a multitude of individual reflectometers (11 microwave bands distributed over the HFS and LFS). These timed bursts are, in turn, used to produce single density profile measurements by means of computationally demanding RT algorithms [6], [7]. Then, an estimation of control plasmawall gaps (separatrix position), at the lines of sight of both HFS and LFS reflectometers, is generated using the locally computed reflectometry profiles, and an estimate of the density at the separatrix obtained from the online measurement of the line-integrated density [1].

Fig. 2 shows a schematic diagram of the PPR and of the plasma position control flow. At ASDEX Upgrade the plasma position feedback loop is operated in a fast $\approx 1 \mathrm{~ms}$ cycle. Due to the configuration of its poloidal field coils, position is controlled separately from plasma shape and hence only the outer radial, $R_{\text {out }}$, and vertical, $Z_{I}$, coordinates of the plasma shape (separatrix) are monitored to calculate the required corrective feedback actuation in the fast control coils [8], [9]. As stated, during the 2011 campaign it was possible to successfully replace the magnetic $R_{\text {out }}$ position satisfying a total latency of $<1 \mathrm{~ms}$, from start of the acquisition of a burst of reflectometry data to the corresponding actuation of the fast coils. This time delay corresponds to the fastest control cycle of the DCS Cycle Master (CM) [5]. Upon receiving from DCS the current control cycle number, the RT diagnostic software waits for all required input data to be available. The PPR depends on the availability of the subscribed linear averaged density, produced by the RT interferometry diagnostic, and on the completion of the reflectometry data DAQ DMA transfer [4]. As soon as all data is available, a multithreaded code preprocesses the reflectometry interference signals, calculates the corresponding electron density profiles and, finally, estimates from them the separatrix position to be fed to the position controller. Because 


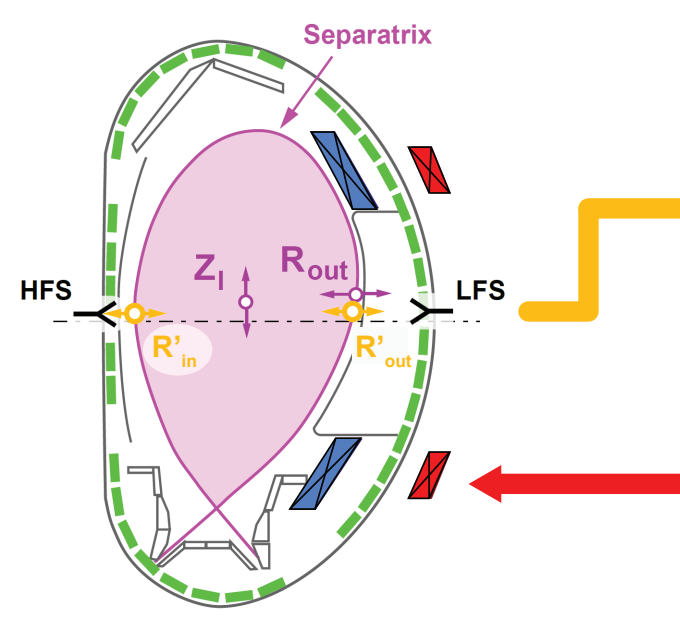

AUG Plasma Position Controller

Input variables: $\mathbf{Z}_{1}$ and $\mathrm{R}_{\text {oUt }} \quad$ (monitored @1 kHz)

\section{RT Reflectometry diagnostic}
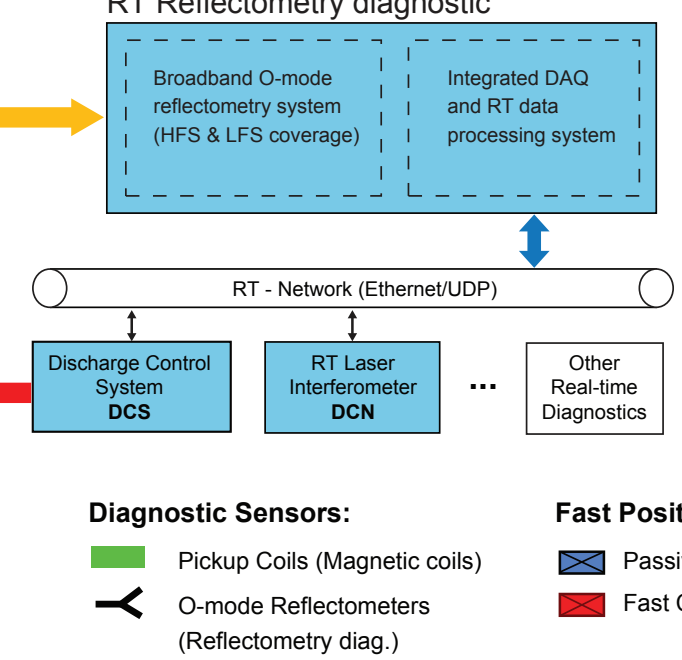

Fast Position Actuators

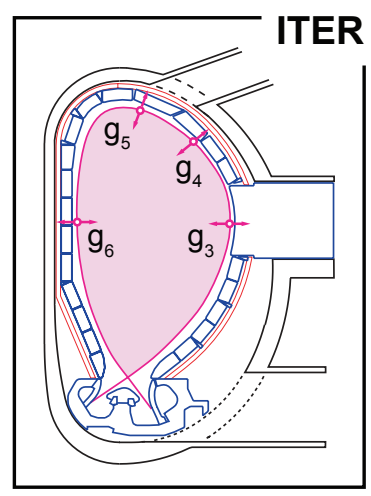

$\varangle$ Passive Saddle Loops

$\square$ Fast Control Coils

Fig. 2. Simplified block diagram of the AUG's PPR control scheme; (inset) ITER PPRs' monitored gaps: $g_{3}-g_{6}$.

not all RT diagnostics produce results in a multiple of the main CM's cycle, no rigid synchronization is imposed to external RT diagnostics such as the PPR. Instead, all RT diagnostics share with the DCS a common synchronized timing source (UTDC board [10]) that is used to timestamp any processed data delivered to the DCS through the RT network (UDP over GbE, in the PPR case). In case all the necessary input data is not available in time at the DCS, a timeout triggers an exception handling mechanism that either replaces the "starved" controller or initiates a different control segment leading to a pulse abortion or a machine soft-landing, for example. During the full feedback demonstration discharges [1] PPR data was always delivered to the DCS well within the maximum latency boundaries, a proof of the deterministic characteristics of the developed RT system.

\section{A. Enhanced RT diagnostic signal coverage}

To increase the reliability and robustness of the diagnostics's HFS and LFS plasma-wall gap estimation and to enable the utilization of all reflectometry channels for RT computations ${ }^{1}$, the existing system was upgraded to allow the acquisition of all available reflectometry signals (13 in all, see Fig. 4). This update extended the covered density range for RT profile computation up to $\approx 6.6 \times 10^{19} \mathrm{~m}^{-3}$, in the HFS, and $\approx 12.4 \times 10^{19} \mathrm{~m}^{-3}$, in the LFS. RT operation of the two $\mathrm{Q}$ and $\mathrm{V} \mathrm{X}$-mode bands will also be possible from now on. When the production of RT profiles using the $\mathrm{V}$ band channels becomes operational (only bands $\mathrm{K}, \mathrm{Ka}$ and $\mathrm{Q}$ are presently used to calculate RT density profiles), it will be possible to directly test the viability of solutions usable in the design of the ITER PPR (DAQ architecture and data processing chain). The acquisition of additional calibration signals used to linearize the oscillator frequency sweep and to detect micro-wave sweeping hardware

\footnotetext{
${ }^{1}$ Previously only HFS and LFS bands K, Ka, Q and V were being acquired for this purpose.
}

anomalies will allow the implementation of RT mechanisms to perform on-line fault detection and recovery. Individual microwave band sweeps take $25 \mu \mathrm{s}$ and are acquired with a 40 MSPS sampling rate (1 Ksample per band and sweep). If needed, the acquisition rate can nevertheless be increased up to 80 MSPS (100 MSPS maximum) doubling in practice the number of samples per sweep, as the duration of the sweep is limited by the installed microwave system hardware. In normal operation, the dual DAQ board system generates twice as much data as before (DMA transfers upload to the hosts always 8 channels worth of data), i.e. $128 \mathrm{~KB}$ of data for each pair of HFS and LFS density profiles.

\section{B. Improved operation mode}

To mimic the way in which the ITER PPR is to be operated, a similar measurements/acquisition mode was implemented. On ITER, the PPR will need to acquire and dispatch (to the scientific data network) burst profile data at a rate $10 \times$ higher than the one needed to compute RT profiles for control purposes $(100 \mu \mathrm{s}$ vs $1 \mathrm{~ms})$. This very high throughput stream will be used to perform detailed offline analysis of events such as ELMs or the L-H transition. Previously, the AUG PPR single DAQ system only acquired the data needed to produce one RT profile/separatrix estimation on a $1 \mathrm{~ms}$ cycle. Presently, restricted by the same $25 \mu$ s microwave sweep rate and $\approx 1.3 \mathrm{~GB} / \mathrm{s}$ maximum effective DAQ upload bandwidth ( $\approx 2.6 \mathrm{~GB} / \mathrm{s}$ dual board aggregate bandwidth), the upgraded system is capable of a reflectometry profile acquisition rate four times higher than before (now 1 profile can be acquired every $250 \mu$ s for off-line analysis) whilst retaining the same RT measurement rate (1 RT profile/plasma-wall gap estimate per $1 \mathrm{~ms}$ ). In all, the RT data processing system needs to handle $8 \times$ more data than before, roughly equivalent to $80 \%$ 


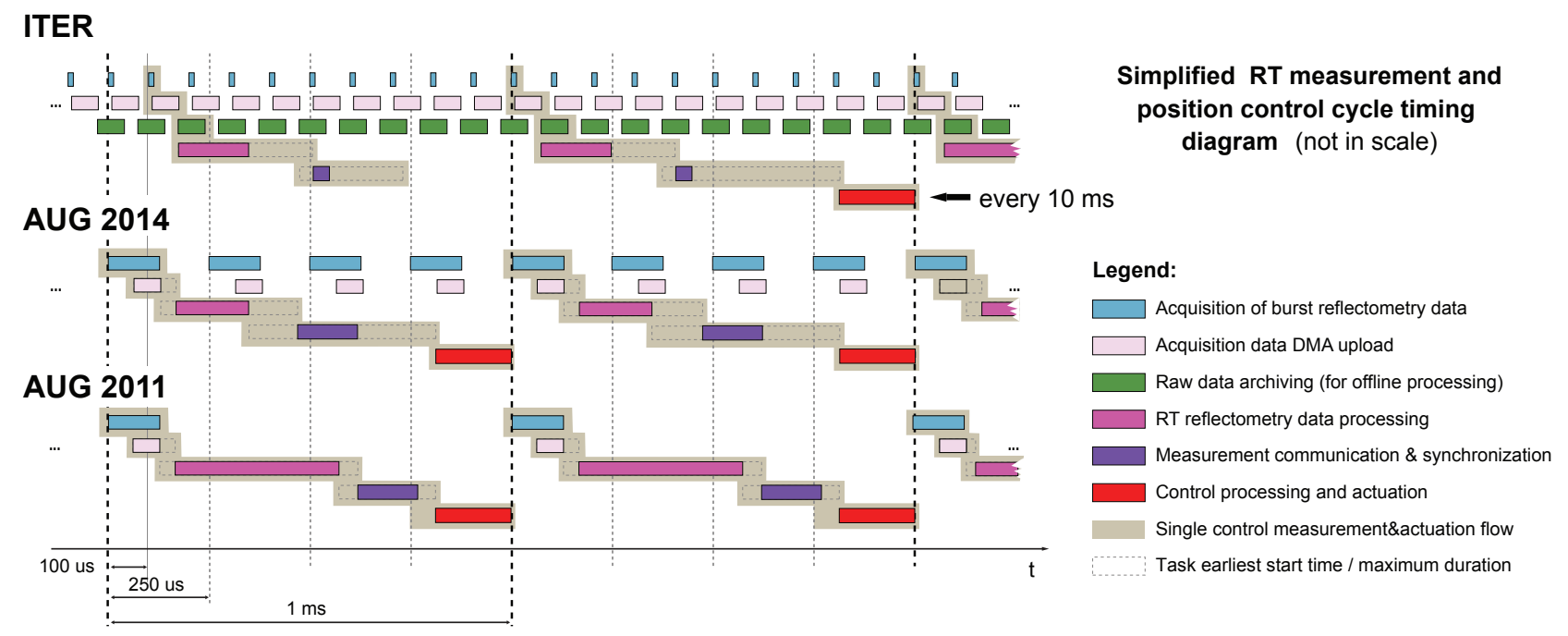

Fig. 3. Diagram of the measurement and RT data processing cycles previously used by the AUG PPR (bottom), presently being implemented on AUG (middle) and planned for ITER PPR (top).

of the expected ITER PPR lowest data bandwidth², without compromising the deterministic characteristics required for the position control application. Fig. 3) shows a timing diagram of the ITER measurement mode, as well as the present and previous modes implemented by the AUG PPR.

\section{UPGRADED SYSTEM}

To achieve the aimed functionality and improved measurement operation mode, system enhancements were made in two fronts. On the hardware side, an additional highbandwidth DAQ board was employed to increase the number of acquisition channels and a modern NUMA based server was chosen to replace the existing host diagnostic server. As the HFS and LFS signals, relevant for the calculation of the control measurements, are acquired in separate DAQ boards, data can be acquired, stored, and processed in each of the two system (NUMA) nodes before it is finally merged and sent to the AUG DCS. On the software side, the RT enabled kernel and custom hardware device drivers, were updated and all the data acquisition and processing chain is being re-written, not only to improve the RAMI characteristics of the system but also to standardize the programming environment, in harmony with all the DCS related software and libraries (now all written in $\mathrm{C}++$ [5]). In the following subsections the choices and developments made in these two fronts are more thoroughly discussed.

\section{A. Hardware improvements}

The need to accommodate in the system a second highbandwidth DAQ board [4] led to a careful choice of the hosting server motherboard architecture. Apart from a needed boost in the computation performance, provided by a dual octo-core

\footnotetext{
${ }^{2}$ Preliminary ITER PPR lowest measurement specification: 8 channels, 1 Ksample sweep data frames, 4 sweeps per profile measurement, 1 offline profile measurement every $100 \mu \mathrm{s}$.
}

configuration (twice the number of cores previously available), the new system's architecture should allow the acquisition and RT processing of data from each of the boards to be performed in two completely separate data flows. The chosen motherboard configuration, a Supermicro MBD-X9DRH-iTF, features two NUMA nodes each with a $2.6 \mathrm{GHz}$ octo-core Intel Xeon E5-2670 and $16 \mathrm{~GB}$ of $1600 \mathrm{MHz}$ DDR3 ECC registered RAM, interconnected by two $8 \mathrm{GT} / \mathrm{s} \mathrm{QPI}^{3}$ links.

Moving from a "Hapertown" (Intel Xeon X5450) to an "Ivy Bridge" based architecture removed the main bottleneck of the former as can be schematically seen in Fig. 4. In fact "Ivy Bridge" CPUs interface directly to their own segregated PCIe bus/devices and possess internal memory management units, directly interfacing to their own memory banks. This arrangement allows for direct DMA transfer between each of the acquisition boards, either HFS or LFS's, and the memory nodes in which data will be processed by the corresponding CPU/attributed cores (magenta and red dashed lines in Fig. 4 for Level 0 and Level 1 processing, respectively). All hardware inter-connections are now much faster and higher than the maximum effective bandwidth of the data acquisition DMA transfer. The system implements a PCIe payload of 256 bytes (previously 128 bytes) which improves the effective DMA bandwidth attainable with the DAQ's PCIe $1.18 \mathrm{x}$ interface. The enhanced measured $\approx 1.3 \mathrm{~GB} / \mathrm{s}$ DMA transfers are easily matched by the native PCIe $3.08 \mathrm{x}$ bus. The system memory interface reaches $80-100 \mathrm{~GB} / \mathrm{s}$, that is $10 \mathrm{x}$ faster than the original memory bandwidth shared between the previous server CPUs through the Northbridge. Furthermore, at the end of the processing chain, when results from both sides (HFS and LFS) will be merged and sent to the DCS by a single thread, access to the non local memory node will be made via dual QPI links that have an aggregated unidirectional bandwidth of $32 \mathrm{~GB} / \mathrm{s}$. In what concerns data transfer no real/practical bottlenecks exist at the hardware architecture

\footnotetext{
${ }^{3}$ Quick Path Interconnect.
} 


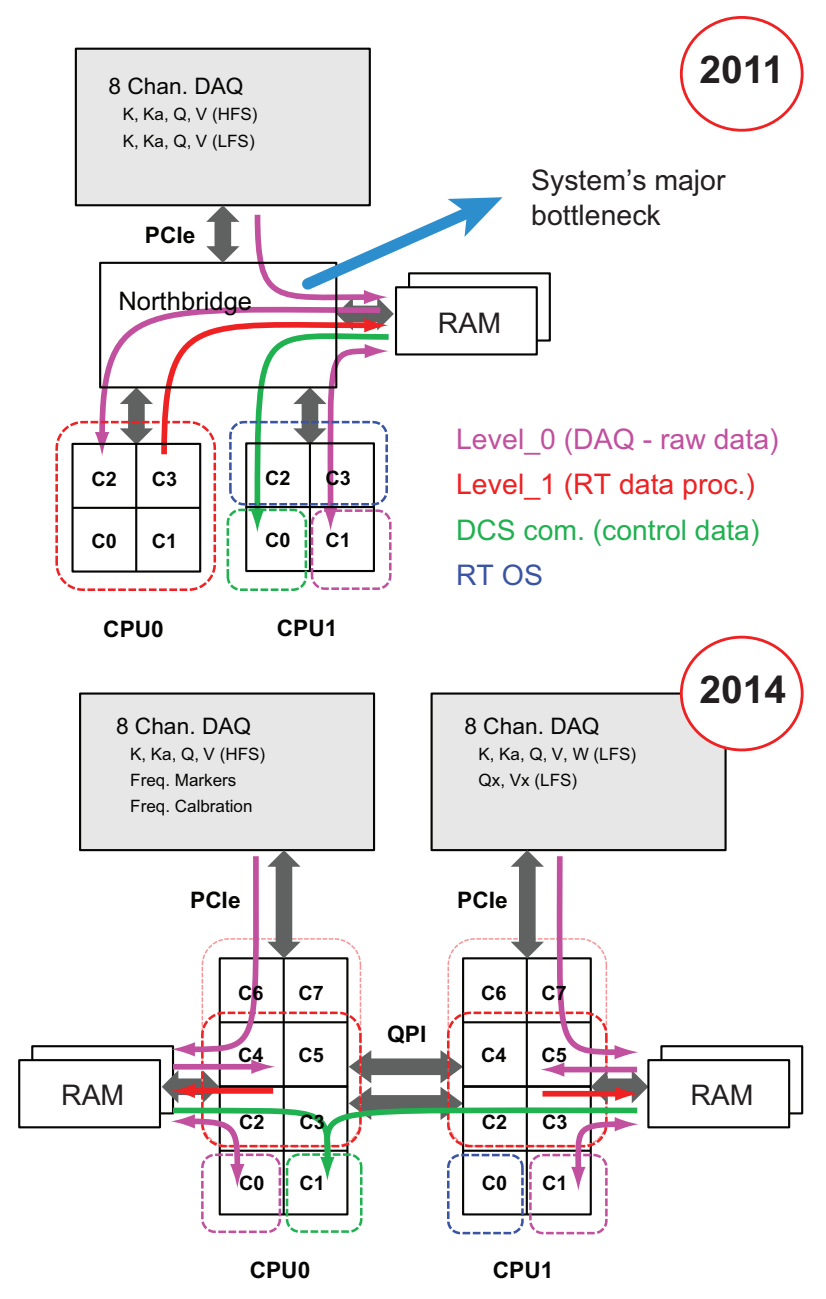

Fig. 4. (top) Simplified block diagram of the RT diagnostic server architecture and DAQ used for the 2011 control demonstration; (bottom) NUMA architecture and DAQ arrangement of the upgraded system used in the present experimental campaign (2014).

level for the aimed application. Additionally, the use of CPU cache was improved in the latter CPU architecture. In place of two L2 $6 \mathrm{MB}$ banks shared between each pair of cores inside each CPU, all cores now feature an individual 256 kB L2 cache and share a single L3 $20 \mathrm{MB}$ cache. This arrangement highly contributes for a more symmetrical and scalable performance of the multithreaded codes handling the RT data processing tasks. "Ivy bridge" CPUs also feature AVX 256 bit SIMD instructions that together with their more optimized architecture implementation compensate the lower clock speed of the chosen CPU configuration (E5-E2670@2.6 $\mathrm{GHz}$ vs. X5450@3.0 GHz). The separated concurrent RT processing of both HFS and LFS density profiles now takes less than half of the time it took before [4] $(\approx 150 \mu \mathrm{s}$ vs $\approx 360 \mu$ s on average) with the same number of cores (4). If needed, 2 extra cores can further be allocated for this purpose (6 per CPU/side, see Fig. 4). Fig. 5 shows pictures of the new RT diagnostic hardware, built using a Supermicro barebone server case and incorporating the custom built, COTS based, DAQ system.

\section{B. Software improvements}

To maintain compatibility with the DCS software infrastructure a modern up-to-date Linux distribution was installed: OpenSUSE 13.1 with a RT patched kernel (version 3.12.15rt25). Moving from a 2.6 kernel to a newer 3.12 kernel involved the adaptation of the DAQ and UTDC hardware device drivers. The main changes, however, were introduced in the main data flow, affecting all data processing phases. Triggered by the adoption of a NUMA based architecture, and by the standardization of $\mathrm{C}++$ as the language of choice for all the DCS related software [5], the complete data processing chain is being re-written from ground up to reflect the separation of HFS and LFS data flows until the very last step, i.e. the estimation of the plasma-wall gaps and their communication to the DCS. Three separate levels have been identified and are now implemented as independent tasks or modules, running in segregated cores (cpusets):

1) Level 0 - DAQ control and raw data gathering and local buffering.

2) Level 1 / RT density profile calculation - Calculation of reflectometry density profiles in RT.

3) Level 1 /CTRL - RT Calculation of plasma-wall gaps for position/shape feedback control, using the online linear integrated density provided by the DCS.

All three levels produce their own AUG official shotfiles and can be activated incrementally. The system can either be used to simply acquire raw data for offline data processing, to acquire raw data and produce RT density profiles (both available immediately after the discharge) or, when connected to the DCS, to additionally produce RT gap data for control purposes. Fig. 6 represents this modular approach and the separation of the software threads to be mapped in both systems' NUMA nodes. This modular implementation is more flexible, easily configurable, maintainable and fine-tunable to the servers specific hardware characteristics. The interprocess communication and synchronization mechanism was also standardized along the data processing chain. The core data is still exchanged via shared memory blocks but task synchronization now exclusively uses counting semaphores.

Improved fault tolerance mechanisms have been implemented, making use of DAQ internal hardware mechanisms (internal timers and burst data timestamping and burst id tagging) and of the new semaphore based process synchronization, to better handle system hiccups, minimizing the impact of such events on the RT measurement stream delivered to the DCS and avoiding potential controller starvation situations. As mentioned before, all blocks still written in C language, such as the module functionality implemented using the previous RT diagnostic framework [11], are also being rewritten in $\mathrm{C}++$ to improve the software maintainability and the reusability of common parts along the complete RT data processing chain.

\section{CONCLUSion}

The described system is presently in the test and commissioning phase on ASDEX Upgrade. Preliminary benchmarks have shown that the goals set for the upgrade have been essentially met. System tuning at the software level 

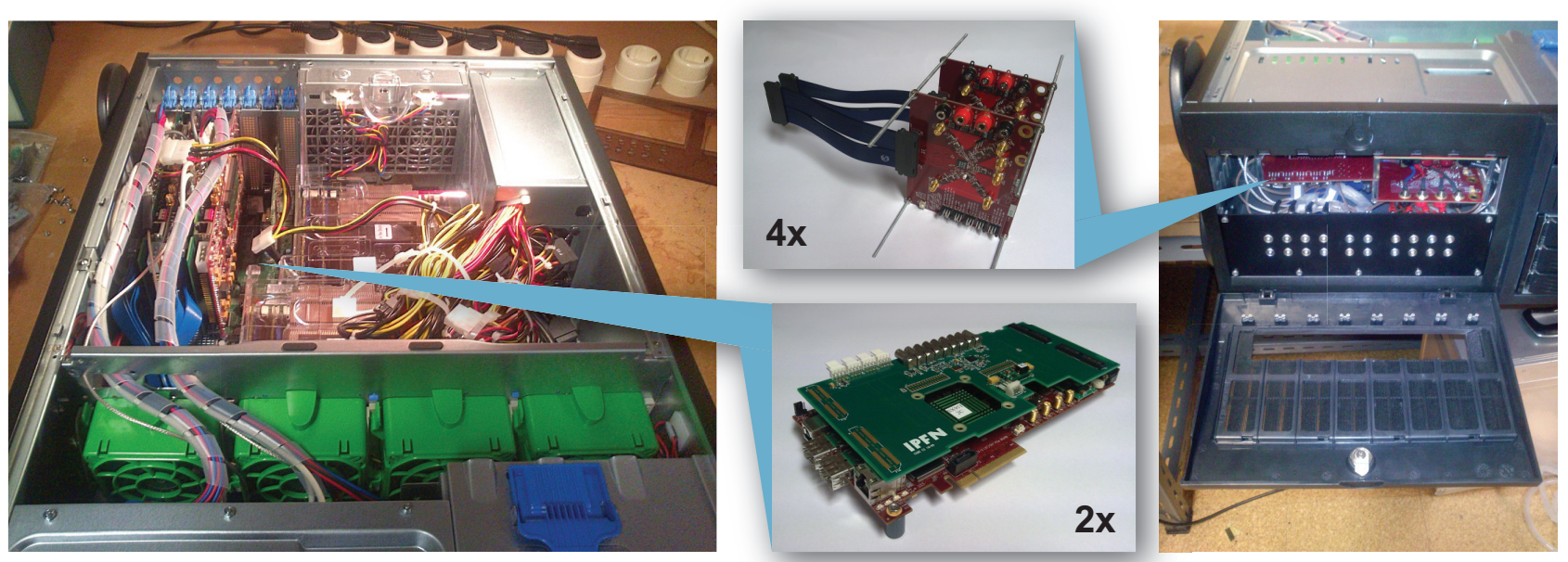

Fig. 5. Photos of the new compact PPR RT data acquisition and processing system.

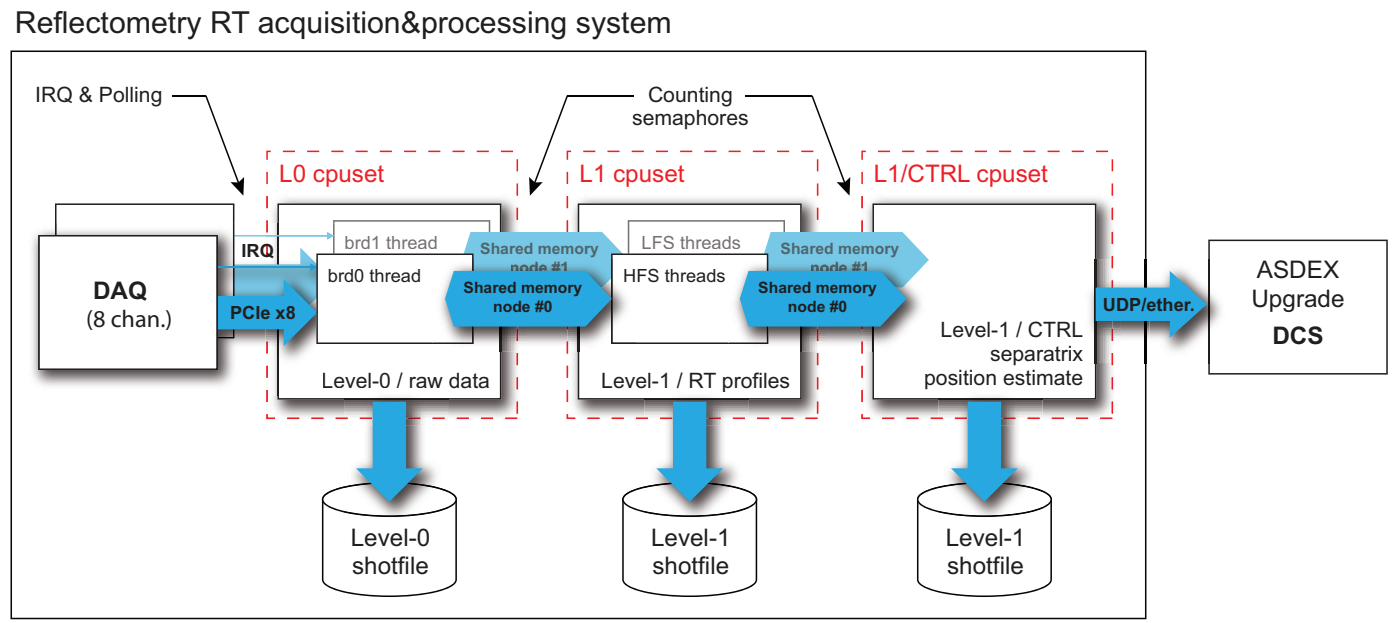

Fig. 6. Block diagram of the RT data processing chain.

is underway to improve the deterministic response of the system in preparation for a feedback control demonstration of the simultaneous use of both HFS and LFS reflectometry data during the 2014 experimental campaign. The experience gained from a successful operation of the control scheme implemented using the herein described DAQ and RT data processing system will play a major role during the design phase of the ITER PPR presently underway.

\section{ACKNOWLEDGMENT}

This work was supported by EURATOM and carried out within the framework of the European Fusion Development Agreement. IST activities also received financial support from Fundação para a Ciência e Tecnologia through project PestOE/SADG/LA0010/2013. The views and opinions expressed herein do not necessarily reflect those of the European Commission. This project has received funding from the EURATOM research and training programme 2014-2018.

\section{REFERENCES}

[1] J. Santos, et al., Reflectometry-based plasma position feedback control demonstration at ASDEX Upgrade, Nuclear Fusion, 52(3), pp 032003.(2012), doi:10.1088/0029-5515/52/3/032003

[2] A. Silva, et al., Microwave reflectometry diagnostic for density profile and fluctuation measurements on ASDEX Upgrade, Rev. Sci. Instrum., 70 (1), pp 1072-1075 (1999)

[3] G. Vayakis G, et al, Status and prospects for mm-wave reflectometry in ITER, Nuclear Fusion, 46 (9), pp. S836-S845 (2006), doi:10.1088/0029$5515 / 46 / 9 / \mathrm{S} 20$

[4] J. Santos, et al, COTS-Based High-Data-Throughput Acquisition System for a Real-Time Reflectometry Diagnostic, IEEE Trans. Nucl. Sci. n, 58, pp. 17518 (2011), doi:10.1109/TNS.2011.2143428

[5] W. Treutterer, et al., ASDEX Upgrade Discharge Control SystemA real-time plasma control framework, Fusion Eng. Des. (2014), doi:10.1016/j.fusengdes.2014.01.001

[6] J. Santos, Reflectometry measurements for plasma postion control purposes, $\mathrm{PhD}$. Thesis, Instituto Superior Técnico, Lisboa, Portugal (2008)

[7] J. Santos, Real-time reflectometry measurement validation in H-mode regimes for plasma position control, Rev. Sci. Instrum. , 81 (10), pp. 10D926 (2010), doi:10.1063/1.3499640

[8] W. Treutterer, et al., Plasma shape control design in ASDEX Upgrade, Proceedings 19th SOFT, (Lisbon, Portugal), vol 1, pp. 933936, Elsevier (1997)

[9] W. Treutterer, et al., ASDEX Upgrades new plasma 
control scheme, Fusion Eng. Des., 81, pp 192731 (2006), doi:10.1016/j.fusengdes.2006.04.010

[10] A. Lohs, et al., The ASDEX Upgrade UTDC and DIO cards - a family of $P C I / c P C I$ devices for real-time DAQ under Solaris, Fusion Engineering and Design, 81, pp 1859-1862 (2006)

[11] M. Reich, et al., Real-time diagnostics and their applications at ASDEX Upgrade, Fus. Sci. Techn., 58 (3), pp. 727-732 (2010) 